11

\title{
Фазовый переход полупроводник-суперионик в пленках сульфида серебра
}

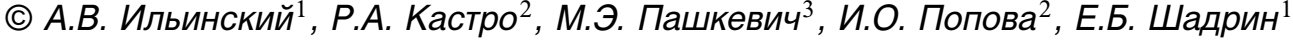 \\ ${ }^{1}$ Физико-технический институт им. А.Ф. Иофрфе РАН, \\ Санкт-Петербург, Россия \\ ${ }^{2}$ Российский государственный педагогический университет им. А. И. Герцена, \\ Санкт-Петербург, Россия \\ ${ }^{3}$ Санкт-Петербургский политехнический университет Петра Великого, \\ Санкт-Петербург, Россия \\ E-mail: shadr.solid@mail.ioffe.ru
}

Поступила в Редакцию 13 июля 2020 г.

В окончательной редакции 13 июля 2020 г.

Принята к публикации 2 августа 2020 г.

\begin{abstract}
Исследованы частотные зависимости тангенса угла диэлектрических потерь $\operatorname{tg} \delta(f)$ и Коул-Коул-диаграммы тонких пленок сульфида серебра при различных температурах в диапазоне $0-200^{\circ} \mathrm{C}$. Проведено сравнение с экспериментальными данными расчета комплексного импеданса предложенной электрической схемы для $\mathrm{Ag}_{2} \mathrm{~S}$-содержащих образцов до и после температурного фазового перехода полупроводник-суперионик. На основе анализа полученных результатов и литературных данных предложена микроскопическая модель фазового перехода в $\mathrm{Ag}_{2} \mathrm{~S}$.
\end{abstract}

Ключевые слова: пленки $\mathrm{Ag}_{2} \mathrm{~S}$, суперионики, фазовый переход, диэлектрическая спектроскопия, атомносиловая микроскопия.

DOI: 10.21883/FTT.2020.12.50218.152

\section{1. Введение}

Процесс изучения материалов с суперионным фазовым переходом (ФП) переживает в последние годы второе рождение в связи с тем, что они интересны и в фундаментальном, и в прикладном отношениях.

Что касается фундаментального аспекта проблемы, то в самое последнее время на основе расчетов из первопринципов и анализа симметрии кристаллической структуры показано, что монокристалл $\mathrm{Ag}_{2} \mathrm{~S}$ с моноклинной симметрией решетки (обозначение Шенфлиса $C_{2}$, обозначение Германа-Могена $\left.P 2_{1} 2_{1} 2_{1}\right)$ является топологическим полуметаллом Дирака [1]. В этом материале сильное спин-орбитальное взаимодействию способствует, согласно [1], появлению между парами Вейля, на которые разбивается каждая точка Дирака, длинных ферми-дуг на поверхностях нанокристаллитов пленки $\mathrm{Ag}_{2} \mathrm{~S}$. Эти образования, как оказалось, устойчивы к механической деформации и воздействию давления, порождаемого поверхностным натяжением нанокристаллитов [1]. Поскольку объемный топологический полуметалл Дирака представляет собой трехмерный кристалл, электроны в котором ведут себя так, как если бы они имели предельно малую массу [2], то подобного рода особенность оказывается актуальной, помимо фундаментального, и в прикладном отношении. А именно, данное свойство материала востребовано в электронике при создании аппаратуры на базе сверхпроводников, а также жестких дисков со сверхвысокой плотностью записи информации. Что касается еще одного аспекта прикладного использо- вания супериоников, иначе называемых твердыми электролитами, то они широко используются как электродные материалы со смешанной электрон-ионной проводимостью, а также как рабочая среда при создании миниатюрных суперконденсаторов сверхбольшой емкости [3].

Наряду с этим, актуальность фундаментального изучения процесса ФП „Полупроводник-суперионик “в тонких нанокристаллических пленках $\mathrm{Ag}_{2} \mathrm{~S}$, обусловлена тем, что для наночастицы суперионный ФП начинается с появления на ее поверхности высокотемпературной неупорядоченной фазы. С ростом температуры граница раздела фаз постепенно движется внутрь наночастицы, однако физический механизм размерной зависимости параметров ФП остается неясным [4].

Интересные результаты при исследовании супериоников дает применение метода диэлектрической спектроскопии (ДС), часто называемой в литературе импедансной спектроскопией [5]. В данном методе исследуются температурные зависимости особенностей функций $\varepsilon_{1}(\omega), \varepsilon_{2}(\omega)$ и $\operatorname{tg} \delta(\omega)$, что для кристаллов $\mathrm{Ag}_{2} \mathrm{~S}$ позволяет с высокой точностью определить энергии активации различных типов ионов $\mathrm{Ag}_{1}^{+}$и $\mathrm{Ag}_{2}^{+}$, имеющих различную координацию [5]. Кроме того, при исследовании пленок супериоников ДС позволяет исследовать размерные зависимости релаксационных параметров нанокристаллитов пленки. Сказанное определяет уникальные возможности метода ДС при изучении супериоников.

Целью работы являлось создание качественной микроскопической модели механизма ФП полупроводник- 


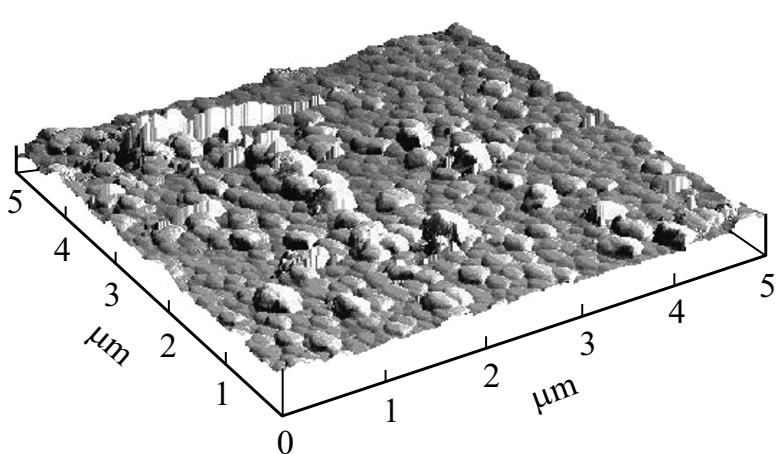

0

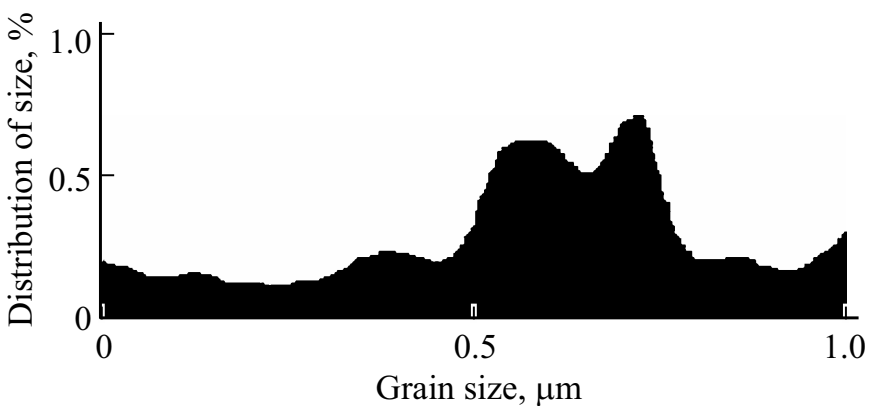

Рис. 1. AFM-изображение пленки $\mathrm{Ag}_{2} \mathrm{~S}$ и гистограмма распределения зерен сульфида серебра по размерам.

суперионик в нанокристалических пленках $\operatorname{Ag}_{2} \mathrm{~S}$. Для достижения этой цели в работе выполнено исследование температурной зависимости диэлектрических спектров пленок $\mathrm{Ag}_{2} \mathrm{~S}$, осуществлено численное моделирование экспериментальных результатов на основе соответствующих эквивалентных схем, а также проведена интерпретация полученных результатов путем сопоставления результатов расчета с параметрами экспериментальных диэлектрических спектров.

\section{2. Образцы и методика эксперимента}

\section{1. Синтез и характеризация нанокристалическх пленок $\mathrm{Ag}_{2} \mathrm{~S}$}

В настоящей работе опытные образцы пленок сульфида серебра были синтезированы двумя методами: термическим сульфидированием слоев металлического серебра в парах серы и химическим осаждением пленок сульфида серебра из раствора солей [6]. При этом на подложках из кристаллов $\mathrm{ZnSe}$ и $\mathrm{BaF}_{2}$ методом лазерной абляции синтезировались тонкие металлические пленки серебра. После обезжиривания в этаноле производилось сульфидирование слоев металлического серебра в кислой среде при температуре $170-180^{\circ} \mathrm{C}$ : использовался $14 \%$ раствор $\mathrm{AgNO}_{3}$ с добавлением водного раствора аммиака и $5 \%$ раствора тиосульфата натрия. Синтезированные пленки были близки по составу к стехиометрическому.

Для регистрации диэлектрических спектров синтезированных образцов пленок $\mathrm{Ag}_{2} \mathrm{~S}$ использовалась планарная методика формирования контактов: на поверхность пленки наносились контактоловые или аквадаговые контакты, расстояние между которыми составляло $5 \mathrm{~mm}$, а площадь контактов составляла $5 \times 15 \mathrm{~mm}^{2}$.

\section{2. Физические параметры синтезированных образцов}

Морфология пленок $\mathrm{Ag}_{2} \mathrm{~S}$ контролировалась путем анализа изображений, полученных с помощью атомно- силового микроскопа (рис 1). Площадь поверхности пленочных образцов составляла: $15 \times 15 \mathrm{~mm}^{2}$, средняя толщина пленок: $2.6 \mu \mathrm{m}$, удельное сопротивление было равно $\rho=7 \cdot 10^{6} \Omega \cdot \mathrm{m}$ при комнатной температуре.

\section{3. Методика ДС}

Измерение диэлектрических спектров и исследования их температурной зависимости выполнялись на диэлектрическом спектрометре марки „Alpha-Beta Impedance Analyzer" фирмы Novocontrol Technologies. Образец пленки $\mathrm{Ag}_{2} \mathrm{~S}$, синтезированной на подложке $\mathrm{BaF}_{2}$, помещался в ячейку спектрометра, электроды которой были соединены с контактами, нанесенными на поверхность пленки. В эксперименте измерялась амплитуда $I_{0}$ тока $\dot{I}(t)$, протекавшего через образец вдоль поверхности пленки при подаче эталонного синусоидального напряжения $U(t)$ амплитудой $1 \mathrm{~V}$. Измерялась также разность фаз $\varphi$ между колебаниями $U(t)$ и $\dot{I}(t)$. С помощью конвертера эти данные преобразовывались в данные о физических характеристиках образца $\left(\operatorname{tg} \delta, \varepsilon^{\prime}, \varepsilon^{\prime \prime}\right)$. Геометрическая емкость пустой ячейки определялась как $\mathrm{C}_{0}$.

Измерения частотных зависимостей $\operatorname{tg} \delta(f), \mathcal{\varepsilon}^{\prime}(f)$, $\varepsilon^{\prime \prime}(f)$ выполнялись в интервале от 10 до $1 \mathrm{MHz}$, температура образца $B$ изменялась в процессе измерений от 0 до $200^{\circ} \mathrm{C}$.

\section{3. Результаты эксперимента}

Диэлектрические спектры (ДС) тонкопленочных нанокристаллических образцов $\mathrm{Ag}_{2} \mathrm{~S}$ представлены на рис. 2 и 3. Рис. 2, $a$ иллюстрирует ДС при сравнительно низкой температуре $T=50^{\circ} \mathrm{C}$, т. е. при температуре существенно ниже температуры $T_{c}=170^{\circ} \mathrm{C}$ суперионного ФП $\left(t<T_{c}\right)$, то есть в фазе моноклинной симметрии. Рис. 2, $b$ представляет ДС при высокой температуре $T=190^{\circ} \mathrm{C}$ (в объемоцентрированной кубической фазе), превышающей температуру $Ф П, T>T_{c}$. На рис. 3 представлены экспериментальные спектры тангенса угла 

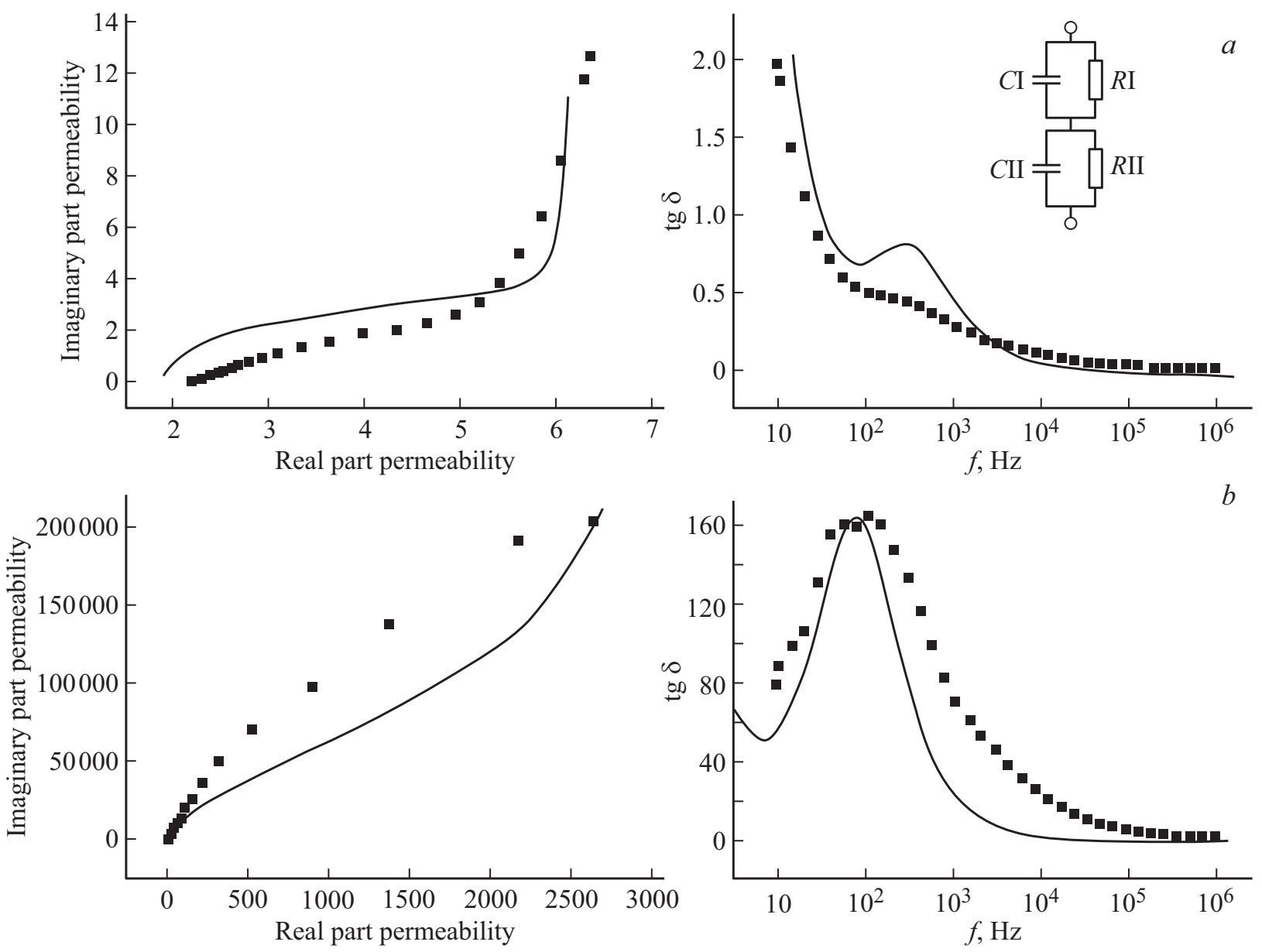

Рис. 2. Коул-Коул-диаграмма и частотные зависимости тангенса угла диэлектрических потерь $\operatorname{tg} \delta(f)$ для пленки $\mathrm{Ag}_{2} \mathrm{~S}$. Точки результат эксперимента, сплошные линии - результат расчета на основе двухзвенной эквивалентной схемы, представленной на вставке к рисунку. $a-$ при температуре $T=50^{\circ} \mathrm{C}, C 0=1.6 \mathrm{pF}, \mathrm{I}=12 \mathrm{pF}, C \mathrm{II}=4 \mathrm{pF}, R \mathrm{I}=600 \mathrm{M} \Omega, R \mathrm{II}=70 \mathrm{M} \Omega . b-$ при температуре $T=190^{\circ} \mathrm{C}, C 0=1.6 . \mathrm{pF}, C \mathrm{I}=4 \mu \mathrm{F}, C \mathrm{II}=35 \mathrm{pF}, R \mathrm{I}=7.5 \mathrm{k} \Omega, R \mathrm{II}=200 \mathrm{k} \Omega$.
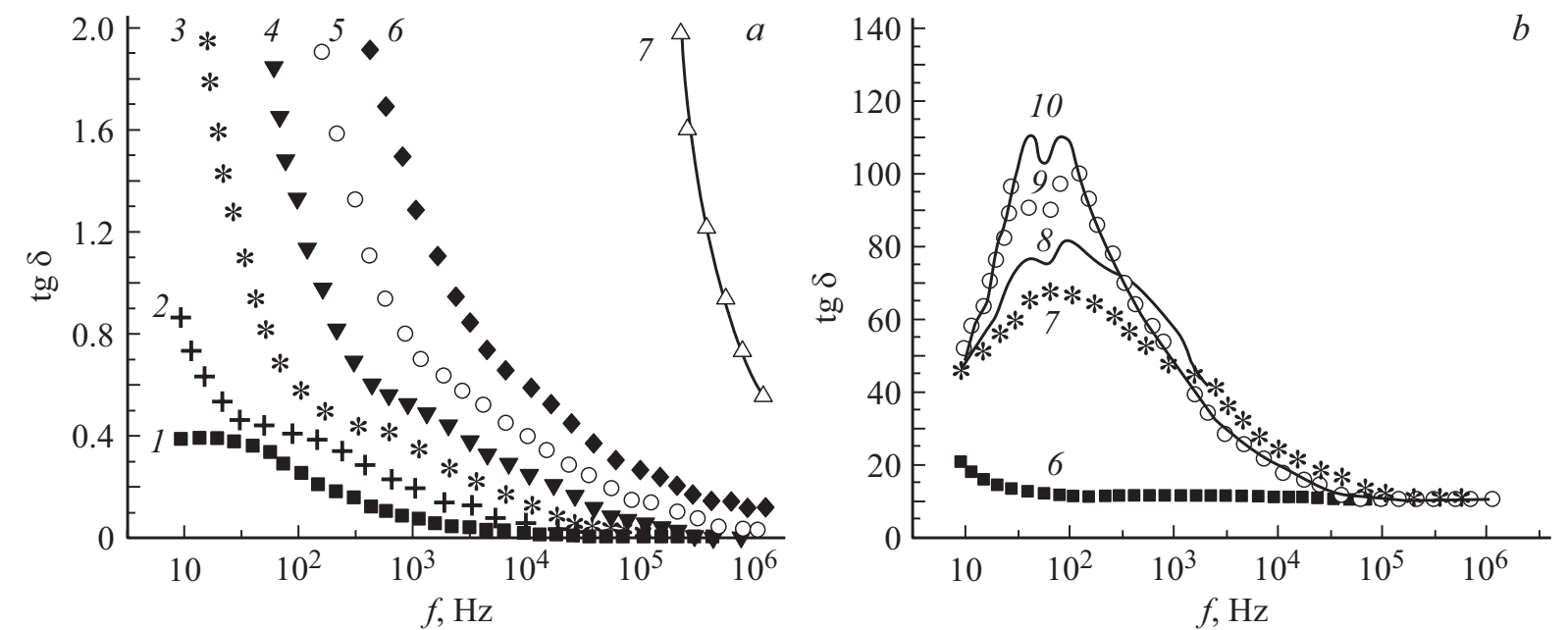

Рис. 3. Частотные зависимости тангенса угла диэлектрических потерь $\operatorname{tg} \delta(f)$ для пленки $\operatorname{Ag}_{2} \mathrm{~S}$ при различных температурах (эксперимент): $1-50,2-70,3-90,4-110,5-130,6-150,7-160,8-170,9-180,10-190^{\circ} \mathrm{C}$. 

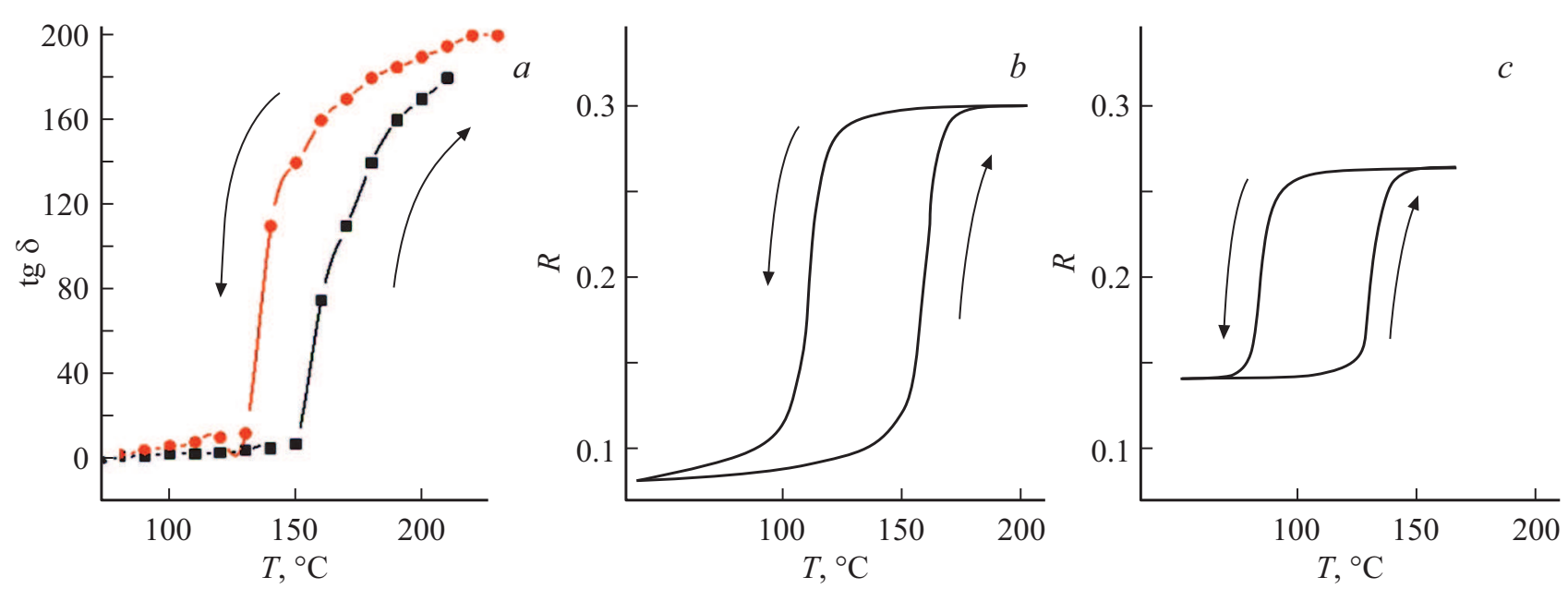

Рис. 4. Петля температурного гистерезиса для пленки $\mathrm{Ag}_{2} \mathrm{~S}$. $a-$ величины максимума $\operatorname{tg} \delta(T)$; $b, c-$ отражательной способности $R$ (зарегистрированной на длине волны $\lambda=7 \mu \mathrm{m}$ ) интерференционной структуры на основе сульфида серебра, полученного сульфидированием пленки металлического серебра на подложке из $\mathrm{BaF}_{2}$ и $\mathrm{ZnSe}$ соответственно.

диэлектрических потерь $(\operatorname{tg} \delta)$ при нескольких промежуточных температурах, полученные при возрастании температуры пленочного образца от 50 до $190^{\circ} \mathrm{C}$.

На рис. $4, a$ представлена температурная зависимость величины $\operatorname{tg} \delta$ в максимуме частотной зависимости, полученная из данных, соответствующих рис. 3. Измерения проводились как при нагреве, так и при охлаждении пленочного образца с целью выявления гистерезисных свойств материала.

Кроме того, на рис. $4, b$ и $4, c$ приведены для сравнения характерные петли температурного гистерезиса отражательной способности интерференционных структур на основе пленки $\mathrm{Ag}_{2} \mathrm{~S}$, нанесенной на $\mathrm{Al}$ металлический подслой, использованный в качестве глухого зеркала интерферометра.

\section{4. Обсуждение результатов}

\section{1. Общие положения}

Прежде всего укажем на то, что на всех спектрах рис. $2-4$ тангенс угла диэлектрических потерь $(\operatorname{tg} \delta)$ либо $>1$ либо $\gg 1$. Это означает, что „омическая“ составляющая переменного тока превышает емкостную, т. е. ток дрейфа носителей превышает ток смещения. Это вынуждает включить в эквивалентную электрическую схему, описывающую электрический отклик образца пленки, активное сопротивление $R$. Кроме того, наличие максимума на частотной зависимости $\operatorname{tg} \delta(\omega)$ говорит о том, что для эквивалентной схемы нельзя обойтись простейшими дифференцирующей или интегрирующей цепочками. На вставке рис. 2 приведена предлагаемая в настоящей работе трехзвенная эквивалентная схема. Ее физический смысл таков: $C$ II и $R \mathrm{II}-$ емкость и сопротивление пленки, $C \mathrm{I}, R \mathrm{I}, C \mathrm{III}, R \mathrm{III}$ суть электрические характеристики приэлектродных областей. Детальный анализ подобного рода эквивалентной схемы приведен ниже.

\section{2. Петли гистерезиса}

Рис. 4 показывает, что представленные на них петли гистерезиса хорошо коррелируют между собой и соответствуют температуре ФП полупроводник-суперионик $T_{c}=152^{\circ} \mathrm{C}$. В дополнение к этому наличие петли температурного гистерезиса также убедительно свидетельствует о том, что в пленках $\mathrm{Ag}_{2} \mathrm{~S}$ в области $T>150^{\circ} \mathrm{C}$ происходит ФП. Особенностью данного ФП является то, что все численные значения величин $\operatorname{tg} \delta, \varepsilon^{\prime}(\omega)$ и $\varepsilon^{\prime \prime}(\omega)$ меняются после совершения ФП на несколько порядков величины.

Причина появления петель гистерезиса заключена, по нашему мнению, в следующем. Согласно литературным данным [4], полученным при исследовании нанокристаллических пленок $\mathrm{Ag}_{2} \mathrm{~S}$ с помощью просвечивающего электронного микроскопа (Transmittance Electron Microscope: TEM) с атомным разрешением, процесс совершения ФП в наночастицах $\mathrm{Ag}_{2} \mathrm{~S}$ различного размера происходит различным образом. А именно, начальная температура появления неупорядоченной фазы для наночастиц малого размера оказывается ниже, чем у крупных наночастиц. И толщина слоя неупорядоченной фазы у малых наночастиц больше, чем у крупных.

Как показано на рис. 1, где приведено AFM-изображение пленки $\mathrm{Ag}_{2} \mathrm{~S}$ и гистограмма распределения нанокристаллитов по размерам, разброс поперечных размеров нанокристаллитов значителен (полуширина гистограммы $0.25 \mu \mathrm{m}$ ). Это, согласно выводам [4], неизбежно должно приводить к возникновению температурного гистерезиса, что и наблюдается в наших экспериментах. Дело в том, что при суперионном ФП изменяется не только точечная симметрия элементарной ячейки 
с моноклинной на объемоцентричную кубическую, но и ее геомерческие размеры. А это связано с необходимостью дополнительных энергетических затрат по принудительному перемещению ионов решетки в новые устойчивые положения с преодолением соответствующих энергетических барьеров [7]. Сказанное справедливо как при переходе из низкотемпературной фазы в высокотемператрную, так и наоборот. Поэтому для совершения ФП требуется отступление по температуре от точки $T_{c}$ равновесия фаз для введения дополнительной тепловой энергии в решетку, что и порождает термический гистерезис. Различие величины отступления от температуры $T_{c}$ равновесия фаз для кристаллитов разного размера связано с вмешательством в энергетику ФП давления, порождаемого, согласно теореме Лапласа, поверхностным натяжением кристаллита. А оно, в свою очередь, обратно пропорционально квадратному корню из среднего поперечника кристаллита. Заметим, что упругая энергия поверхностного натяжения может не только мешать совершению ФП, но и способствовать ему в зависимости от того, рассеивается ли полностью запасенная после синтеза упругая энергия кристаллита или нет.

Согласно данным [7], расстояния между ионами серы высокотемпературной фазы меньше, чем низкотемпературной. Объем элементарной ячейки при суперионном ФП в высокотемпературную фазу уменьшается вдвое [8]. Это автоматически приводит к тому, что лапласовское поверхностное давление способствует переходу в высокотемпературную фазу, то есть в мелких нанокристаллитах $T_{c}+\Delta T(\Delta T-$ отступление по температуре, необходимое для совершения ФП в кристаллите данного размера) должна быть ниже, чем в крупных, что и наблюдалось экспериментально в [4].

Дополнительное подтверждение данного тезиса присутствует на рис. 4, $b$, где показан протяженный по температуре „хвост“ в низкотемпературной части петли гистерезиса. Дело в том, что петли на рис. $4, b$ и рис. 4, $c$ получены для пленок $\mathrm{Ag}_{2} \mathrm{~S}$, синтезированных на различных подложках: рис. $4, b-$ на $\mathrm{BaF}_{2}$, рис. $4, c-$ на ZnSe. Это говорит о вмешательстве в энергетику ФП упругой энергии взаимодействия нанокристаллитов $\mathrm{Ag}_{2} \mathrm{~S}$ с подложкой при их адгезии к ней в процессе синтеза, которая резко различается для $\mathrm{BaF}_{2}$ и $\mathrm{ZnSe}$ виду различий степени несовпадения постоянных кристаллической решетки $\mathrm{Ag}_{2} \mathrm{~S}$ и постоянных кристаллической решетки подложек. Здесь также принципиальным является способность к рассеиванию адгезионной упругой энергии, которая, как и в случае энергии поверхностного натяжения, может либо мешать либо способствовать совершению ФП. Заметим, что толщина металлического подслоя (нижнего зеркала) интерференционной структуры была порядка скин-слоя металла Al (300 А), что эффективно декорировало структуру поверхности подложки, не внося искажений в упомянутое несовпадение постоянных решетки и, тем самым, в значения упругих характеристик адгезионного взаимодействия нанокристаллитов $\mathrm{Ag}_{2} \mathrm{~S}$ с подложкой.

На базе процедуры минимизации свободной энергии поверхности и межфазной свободной энергии нанокристаллитов в [4] развита феноменологическая модель, непротиворечивым образом объясняющая наличие размерной зависимости температуры ФП в $\mathrm{Ag}_{2} \mathrm{~S}$. Полученные нами с помощью оптических экспериментальных методов результаты полностью подтверждают выводы упомянутой работы. Дело в том, что при ФП особенности появления новой фазы на поверхности старой сохраняются, как указано, как при повышении так и при понижении температуры, что отражает мартенситный характер ФП и с неизбежностью порождает гистерезис физических параметров пленки $\mathrm{Ag}_{2} \mathrm{~S}$, если существует разброс нанокристаллитов по размерам (рис. 1).

Обратим внимание на петлю гистерезиса величины пика $\operatorname{tg} \delta(\omega)$ пленки $\operatorname{Ag}_{2} \mathrm{~S}$ (рис. 4,a). Ее форма отличается от формы оптических петель (рис. 4, $b, c$ ) существенной асимметрией ветвей. Дело в том, что электрический отклик образца обусловлен, в отличие от оптического отклика, процессом образования бесконечного перколяционного кластера, что отражено в характерном виде ветвей петли: они имеют резкий подъем при достижении перколяционного порога и протяженную по температуре область завершения формирования кластера. Кроме того, форма ветвей петли гистерезиса максимума $\operatorname{tg} \delta(\omega)$ через функции $R(T)$ и $C(T)$ отражает зависимость релаксационных характеристик суперионного ФП от величины лапласовского давления, порождаемого поверхностным натяжением кристаллита, поскольку проводящие и емкостные свойства кристаллита зависят от близости его температуры к $T=T_{c}+\Delta T$. Положение $T=T_{c}+\Delta T$ на температурной шкале управляется, в свою очередь, величиной лапласовского давления, зависящего от размера кристаллита и задающего $\Delta T$. Как показано на рис. 3, максимум кривой $\operatorname{tg} \delta(\omega)$ имеет вблизи $T_{c}$ сложную структуру в виде нескольких слабо разрешенных пиков, что связано, по нашему мнению, со сложной структурой гистограммы распределения кристаллитов пленки по размерам (рис. 1). Кроме того, эксперимент показывает, что форма максимума кривой $\operatorname{tg} \delta(\omega)$ видоизменяется различным образом при изменении температуры вдоль нагревной и охладительной ветвей петли гистерезиса (на рис. 3 изменение кривой при охлаждении не представлено). Это говорит о том, что ферми-дуги между парами Вейля на поверхностях нанокристаллитов пленки $\mathrm{Ag}_{2} \mathrm{~S}$ по-разному реагируют на лапласовское поверхностное давление при образовании вблизи $T_{c}$ зародышей новой фазы. А именно, принципиально важно, сопровождается ли ФП зарождением суперионной фазы в толще полупроводниковой или же, наоборот, зарождением полупроводниковой фазы в толще суперионной. В первом случае с постом температуры сначала будут совершать ФП зерна малых размеров, а после этого - зерна больших размеров; во втором случае при понижении 
температуры, наоборот, сначала совершают ФП крупные зерна, а после этого - зерна малых размеров. Данное обстоятельство, в свою очередь, связано в различием величин энергии, необходимой для трансформации параметров кристаллической решетки при движении вдоль нагревной и охладительной ветвей петли гистерезиса, поскольку при нагреве одни параметры кристаллической ячейки вблизи $T_{c}$ увеличиваются, а другие - уменьшаются. А именно, параметр решетки $a$ увеличивается с $a=0.4231 \mathrm{~nm}$ в моноклинной фазе до $0.4884 \mathrm{~nm} \mathrm{в}$ кубической фазе, а параметры $b$ и $c$ уменьшаются с $b=0.6930 \mathrm{~nm}$ и $c=0.9526 \mathrm{~nm}$ до $b=c=0.4884 \mathrm{~nm}$. При охлаждении, наоборот, параметр решетки $a$ уменьшается, а параметры $b$ и $c$ растут. При этом не вызывает сомнения, что энергетические затраты (то есть $\Delta T)$, необходимые для перемены положений ионов с переходом в высокосимметричную фазу не совпадают с таковыми при переходе в низкосимметричную фазу.

Из сказанного следует, что процесс суперионного ФП в $\mathrm{Ag}_{2} \mathrm{~S}$ нетривиален, причем метод ДС позволяет проследить за тонкими деталями термического изменения физических параметров топологических полуметаллов, которые недоступны другим методам, в чем и состоит уникальность ДС как метода исследования. Подробное изучение описанных явлений должно послужить предметом дальнейших исследований.

\section{3. Расчеты комплексного импеданса сформированной электрической схемы для тонкопленочных $\mathrm{Ag}_{2} \mathrm{~S}$--содержащих образцов}

В качестве эквивалентной схемы изначально была выбрана схема, состоящая из трех последовательно расположенных $R C$-звеньев. В этой схеме параллельно соединенные $C$ I и $R$ I, $C$ II и $R$ II, $C$ III и $R$ III представляют собой соответственно емкость и сопротивление между пленкой и проводящим контактом, нанесенным на пленку $\mathrm{Ag}_{2} \mathrm{~S}$, емкость и сопротивление самой пленки $\mathrm{Ag}_{2} \mathrm{~S}$, емкость и сопротивление между пленкой и вторым контактом. Расчет параметров трехзвенной схемы выполнен нами символическим методом. В такой схеме для частотных зависимостей действительной $\varepsilon^{\prime}(\omega)$ и мнимой $\varepsilon^{\prime \prime}(\omega)$ частей диэлектрической проницаемости пленочного образца получены следующие выражения:

$$
\begin{gathered}
\varepsilon^{\prime}(\omega)=\mathrm{B} /\left[\omega \operatorname{Co}\left(\mathrm{A}^{2}+\mathrm{B}^{2}\right)\right], \\
\varepsilon^{\prime \prime}(\omega)=-\mathrm{A} /\left[\omega \operatorname{Co}\left(\mathrm{A}^{2}+\mathrm{B}^{2}\right)\right], \\
\operatorname{tg} \delta=\varepsilon^{\prime \prime}(\omega) / \varepsilon^{\prime}(\omega),
\end{gathered}
$$

где

$$
\begin{aligned}
\mathrm{A}= & R \mathrm{I} /\left(1+\omega^{2} C \mathrm{I}^{2} R \mathrm{I}^{2}\right)+R \mathrm{II} /\left(1+\omega^{2} C \mathrm{II}^{2} R \mathrm{II}^{2}\right) \\
& +R \mathrm{III} /\left(1+\omega^{2} C \mathrm{III}^{2} R \mathrm{IIII}^{2}\right)
\end{aligned}
$$

$$
\begin{aligned}
\mathrm{B}= & \omega C \mathrm{II}^{2} /\left(1+\omega^{2} C \mathrm{I}^{2} R \mathrm{I}^{2}\right)+\omega C \mathrm{II} R \mathrm{II}^{2} /\left(1+\omega^{2} C \mathrm{II}^{2} R \mathrm{II}^{2}\right) \\
& +\omega C \mathrm{III} R \mathrm{III}^{2} /\left(1+\omega^{2} C \mathrm{III}^{2} R \mathrm{III}^{2}\right) .
\end{aligned}
$$

Эквивалентная схема включает в себя, таким образом, три емкости и три сопротивления, величины которых, как следует из рис. 2,3, сильно зависят от температуры. Однако предполагается, что оба контактоловых или аквадаговых электрических контакта являются симметричными с практически одинаковыми характеристиками по отношению к приложенному синусоидальному напряжению, поскольку постоянное электрическое смещение отсутствует. Поэтому, как показывают выражения (1-3), трехзвенная электрическая схема реально сводится к двухзвенной $(C \mathrm{I}=C \mathrm{III}, R \mathrm{I}=R \mathrm{III})$, приведенной на вставке к рис. 2. Расчетные графики функций $\operatorname{tg} \delta(\omega)$ и $\varepsilon^{\prime \prime}\left(\varepsilon^{\prime}\right)$ приведены на рис. 2,3 сплошными линиями. Эти результаты получены для упрощенной двухзвенной схемы, которая хорошо описывает появление одного максимума на частотной зависимости $\operatorname{tg} \delta(\omega)$, а также вид Коул-Коул-диаграммы, то есть вид графика функции $\varepsilon^{\prime \prime}\left(\varepsilon^{\prime}\right)$. Укажем, что построение $\varepsilon^{\prime \prime}\left(\varepsilon^{\prime}\right)$ осуществлялось в параметрическом виде, причем роль параметра играла частота $\omega$.

Заметим, что $\lim \left(\varepsilon^{\prime \prime}(\omega)\right)=0$ (при $\left.\omega \rightarrow \infty\right)$, при этом положение левой точки $\varepsilon^{\prime}$ (при $\left.\omega \rightarrow \infty\right)$ на КоулКоул-диаграммах рис. 2 не зависит от величин сопротивлений и определяется только значениями емкостей из соотношения $\varepsilon^{\prime}=(C \mathrm{I} \times C \mathrm{II}) /[\mathrm{CO}(C \mathrm{I}+C \mathrm{II})]$. Второй предел $\lim \left(\varepsilon^{\prime \prime}(\omega)\right)=\infty \quad($ при $\omega \rightarrow 0), \quad$ при этом $\varepsilon^{\prime}=\left(C \mathrm{I} \times R \mathrm{I}^{2}+C \mathrm{II} \times R \mathrm{II}^{2}\right) /\left[\mathrm{C} 0(R \mathrm{I}+R \mathrm{II})^{2}\right]$. Полученные выражения облегчают нахождение параметров схемы в результате подгонки расчетных кривых к экспериментальным данным. Найденные параметры приведены в подписях к рис. 2. Анализ рис. 2 показывает, что величины обеих емкостей схемы увеличиваются после совершения суперионного ФП, в то же время оба сопротивления схемы уменьшаются.

Причина такого положения заключена в температурном росте проводимости (то есть уменьшение сопротивления) и накоплении свободного металлического серебра на аквадаговых электродах, неспособных проводить ионный ток, что порождает рост приконтактных емкостей.

\section{4. Микроскопическая модель ФП полупроводник-суперионик в $\mathrm{Ag}_{2} \mathrm{~S}$}

При построении модели ФП мы будем исходить из следующих физических соображений. $\mathrm{Ag}_{2} \mathrm{~S}$ существует в трёх кристаллических модификациях:

$\alpha-\mathrm{Ag}_{2} \mathrm{~S}$, моноклинная сингония, пространственная группа $P 2_{1} / \mathrm{c}, \quad$ параметры ячейки $a=0.4231 \mathrm{~nm}$, $b=0.6930 \mathrm{~nm}, c=0.9526 \mathrm{~nm}, \beta=125.48$. Объем элементарной ячейки $V=0.28 \mathrm{~nm}^{3}, \quad Z=8$, плотность $D=7.317 \mathrm{~g} / \mathrm{sm}^{3}$.

$\beta-\mathrm{Ag}_{2} \mathrm{~S}$, кубическая сингония, пространственная группа $I \mathrm{~m} 3 \mathrm{~m}$, параметры ячейки $a=0.4884 \mathrm{~nm}$. Объем 


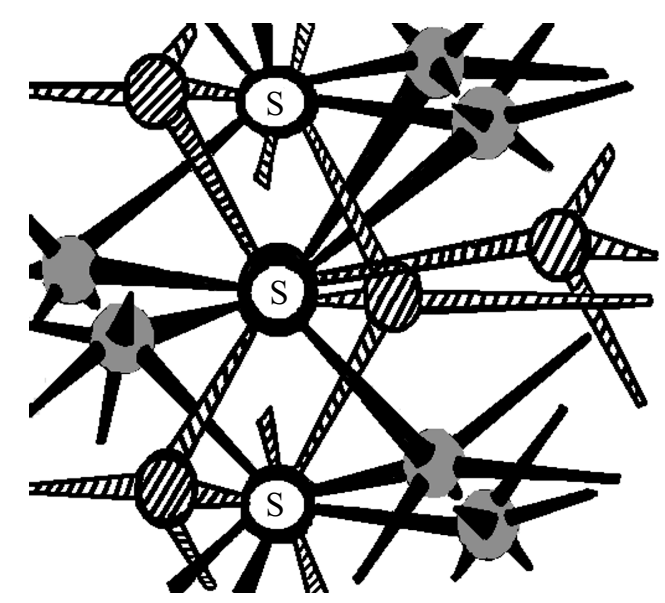

Рис. 5. Фрагмент кристаллической решетки $\mathrm{Ag}_{2} \mathrm{~S}$ : заштрихованные кружки - тетракоординированные ионы $\mathrm{Ag}^{+}$, тонированные кружки - октакоординированные ионы $\mathrm{Ag}^{+}$, свободные кружки - ионы $\mathrm{S}^{2+}$.

элементарой ячейки $V=0.117 \mathrm{~nm}^{3} . Z=2$, плотность $D=7.2 \mathrm{~g} / \mathrm{sm}^{3}$.

Отметим принципиально важное обстоятельство: объем элементарной ячейки при суперионном ФП в выскосимметричную кубическую фазу уменьшается практически вдвое.

$\gamma-\mathrm{Ag}_{2} \mathrm{~S}$, кубическая сингония, пространственная группа $P \mathrm{n} 3$, параметры ячейки $a=0.634 \mathrm{~nm}, Z=4$, плотность $D=6.42 \mathrm{~g} / \mathrm{sm}^{3}$. Температуры ФП в объемном монокристалле: $\alpha \rightarrow \beta 176.3^{\circ} \mathrm{C}, \beta \rightarrow \gamma 592^{\circ} \mathrm{C}$.

Как показывает анализ кристаллической структуры $\mathrm{Ag}_{2} \mathrm{~S}$ (рис. 5), все ионы кристаллической решетки как в высокотемпературной, так и в низкотемпературной фазах $\mathrm{Ag}_{2} \mathrm{~S}$ соединены между собой $\sigma$-связями.

Ионы $\mathrm{S}^{2-}$ непосредственно не образуют $\sigma$-связей между собой. То же относится к ионам $\mathrm{Ag}^{+}$: ионы $\mathrm{Ag}^{+}$также непосредственно между собой не соединены $\sigma$-связями. Ионы $\mathrm{S}^{2-}$ занимают фиксированные положения в кристаллической решетке исключительно благодаря стабилизации их положений через $\sigma$-связи с ионами $\mathrm{Ag}^{+}$.

Для создания полноценных $\sigma$-связей ионов $\mathrm{S}^{2-}$ с 9 ионами $\mathrm{Ag}^{+}$их ближайшего окружения (рис. 5) требуется 18 электронов. При этом атом S, будучи девяти координированным, должен находиться в состоянии гибридизации согласно электронной конфигурации: $1 \mathrm{~s}^{2}(1) 2 \mathrm{~s}^{2}(1) 2 \mathrm{P}_{\mathrm{xy}}^{4}(2)\left\{2 \mathrm{P}_{\mathrm{z}}^{2}(1) 3 \mathrm{~s}^{2}(1) 3 \mathrm{P}_{\mathrm{xy}}^{4}(2) 3 \mathrm{P}_{z}^{0}(1) 4 \mathrm{~s}^{0}(1) 4 \mathrm{P}_{\mathrm{xyz}}^{0}(3)\right\}$. Заключенные в фигурные скобки атомные орбитали создают 9 гибридных орбиталей с 8 электронами на них, позволяющих создать $9 \sigma$-связей с ионами $\mathrm{Ag}^{+}$. Здесь числа в скобках определяют число орбиталей данного типа, верхние индексы - число электронов на них, нижние индексы - пространственную ориентацию орбиталей. Ион $\mathrm{S}^{2-}$ отдает, таким образом, на создание $\sigma$-связей 8 электронов.
Тетра-координированный ион $\mathrm{Ag}^{+}$находится в состоянии гибридизации согласно формуле: $\operatorname{Ar} 3 \mathrm{~d}^{10}(5) 4 \mathrm{~s}^{2}(1) 4 \mathrm{P}^{6}(3) 4 \mathrm{~d}^{6}(3)\left\{4 \mathrm{~d}^{4}(2) 5 \mathrm{~s}^{1}(1) 4 \mathrm{f}^{0}(1)\right\} 4 \mathrm{f}^{0}(6)$ (рис. 5). Заключенные в фигурные скобки атомные орбитали создают 4 гибридные орбитали для создания 4-х $\sigma$-связей с атомами S. На эти 4 орбитали приходится 5 электронов, что в среднем делегирует на каждую орбиталь 5/4 электронной плотности. В ближайшем окружении иона $\mathrm{S}^{2-}$ находится 4 тетра-координированных иона $\mathrm{Ag}^{+}$, которые отдают на создание 4-x S-Ag- $\sigma$-связей в целом 5 электронов.

Окта-координированный ион $\mathrm{Ag}^{+}$находится в состоянии гибридизации согласно конфигурации: $\operatorname{Ar} 3 \mathrm{~d}^{10}(5) 4 \mathrm{~s}^{2}(1) 4 \mathrm{P}^{6}(3) 4 \mathrm{~d}^{4}(2)\left\{4 \mathrm{~d}^{6}(3) 4 \mathrm{f}^{0}(3)\right\} 4 \mathrm{f}^{0}(6) 5 \mathrm{~s}^{1}(1)$.

На эти 6 орбиталей приходится 6 электронов, что в среднем делегирует на каждую орбиталь $(6 / 6)=1$ электронной плотности. В ближайшем окружении иона $\mathrm{S}^{2-}$ находится 5 окта-координированных иона $\mathrm{Ag}^{+}$(рис. 5), которые отдают на создание $5 \mathrm{~S}-\mathrm{Ag}-\sigma$-связей в целом 5 электронов.

Обратим внимание на одно важную особенность атома $\mathrm{Ag}$. А именно, $4 f^{0}(7)$ орбитали этого атома остаются свободными от электронов, а электрон, необходимый для заселения уровней $4 f^{0}(7)$ атома $\mathrm{Ag}$ в соответствии с его положением в таблице химических элементов располагается на следующей атомной орбитали $5 s^{1}(1)$. Это возможно лишь в том случае, если атомная орбиталь $5 s^{1}(1)$ оказывается после своего заселения электроном ниже по энергии $4 \mathrm{f}^{0}(7)$-орбитали. Отсюда следует, что такой элемент, как $\mathrm{Ag}$, является одним из сильно коррелированных элементов, у которых энергетическое положение атомных уровней зависит от их заселенности электронами. И, кроме того, отсюда следует, что энергии $4 \mathrm{f}^{0}(7)$ и $5 \mathrm{~s}^{1}(1)$ атомных орбиталей близки друг к другу. А это означает, что указанные орбитали могут на равных условиях участвовать в формировании гибридных орбиталей, что, согласно теории молекулярных орбиталей, является разрешенной процедурой [9].

В общем итоге от ионов $\mathrm{Ag}^{+}$получено $5+5=10$ электронов, которые вместе с 8 электронами иона $\mathrm{S}^{2-}$ позволяют образовать 9 полноценных S-Ag- $\sigma$-связей (по 2 электрона на каждую связь). Говоря более детально, укажем, что 5 окта-координированных ионов $\mathrm{Ag}^{+}$отдают на $\sigma$-связи каждый по 1 электрону. Также поступает и ион $\mathrm{S}^{2-}$, отдавая 5 электронов на эти связи: по 1 электрону на каждую связь.

Поэтому в распоряжении иона $\mathrm{S}^{2-}$ остается для создания 4-х $\sigma$-связей с 4-мя тетра-координированными ионами $\mathrm{Ag}^{+}$только 3 электрона из 8. То есть ион $\mathrm{S}^{2-}$ на каждую из 4-х тетра-Ag-S- $\sigma$-связей способен отдать в среднем лишь по 3/7 электронной плотности. Недостаток плотности восполняется тем, что каждый тетра-координированный ион $\mathrm{Ag}^{+}$отдает на каждую гибридную орбиталь $5 / 4$ электронной плотности, что вместе с $3 / 4$ электронной плотности иона $\mathrm{S}^{2-}$ позволяет сформировать 4 полноценные $\sigma$-связи с 2 электронами на каждую связь. 
Из сказанного следует, что каждая из 4-х тетра$\mathrm{Ag}-\mathrm{S}-\sigma$-связей не является полностью равноценной по отношению к каждой из 5-ти окта-Ag-S- $\sigma$-связей. Это происходит ввиду того, что степень владения участниками $\sigma$-связей обобществленной парой электронов определяется различием в электроотрицательности атомов $\mathrm{S}$ и Ag. Электроотрицательность атома $\mathrm{S}$ почти в полтора раза выше электроотрицательности атома Ag (величины относительных электроотрицательностей соответственно 2.58 для $\mathrm{S}$ против 1.9 для $\mathrm{Ag}$; абсолютные величины: 5.77 для $\mathrm{S}$ против 4.25 для $\mathrm{Ag}[10])$. В результате в окта$\mathrm{Ag-S}-\sigma$-связи суммарная электронная плотность пары электронов сильно сдвинута в сторону иона $\mathrm{S}^{2-}$. В то же время в тетра-Ag-S- $\sigma$-связи суммарная электронная плотность несколько отодвинута от иона $\mathrm{S}^{2-}$, будучи смещенной в сторону центра $\sigma$-связи, поскольку здесь атом $\mathrm{S}$ отдает на связь лишь $3 / 4$ электронной плотности, тогда как атом Ag отдает 5/4 электронной плотности.

Отсюда, согласно теории молекулярных орбиталей, непосредственно следует, что энергии связей и степень перекрытия орбиталей в них должны различаться. А поскольку, согласно общим положениям квантовой химии, чисто ковалентные связи (электронная плотность в центре связи) являются более прочными, нежели связи с высокой степенью ионности (электронная плотность сдвинута в сторону одного из атомов) [11], то энергия тетра-Ag-S- $\sigma$-связи выше энергии окта-Ag-S- $\sigma$-связи [9]. При этом тетра-Ag-S- $\sigma$-связи в среднем короче окта$\mathrm{Ag}-\mathrm{S}-\sigma$-связей (рис. 5) ввиду большего перекрытия их гибридных орбиталей.

Из этого, в свою очередь, следует, что с ростом температуры в первую очередь происходит разрыв окта$\mathrm{Ag}-\mathrm{S}-\sigma$-связей решетки $\mathrm{Ag}_{2} \mathrm{~S}$, то есть ионы окта- $\mathrm{Ag}^{+}$ при меньшей температуре, чем тетра- $\mathrm{Ag}^{+}$получают возможность скачкообразно двигаться по кристаллической решетке подобно жидкости с высокой степенью вязкости.

После разрыва менее прочных $\mathrm{Ag}-\mathrm{S}$-связей кристаллическая решетка остается стабилизированной более прочными тетра-Ag-S- $\sigma$-связями, хотя и меняет свою симметрию с моноклинной на кубическую под воздействием сил кристаллического каркаса (рис. 5) [7]. Описанный процесс представляет собой процесс ФП „Полупроводник-суперионик“.

Обратим внимание на одно принципиально важное обстоятельство. А именно, низкотемпературная фаза $\mathrm{Ag}_{2} \mathrm{~S}$ представляет собой прямозонный полупроводник с шириной запрещенной зоны $0.9 \mathrm{eV}$ [12]. При этом если в молекуле типичная энергия химической связи с большой степенью ионости составляет $4 \mathrm{~J} / \mathrm{mol}$ (или около $4 \mathrm{eV}$ ), а энергия чисто ковалентной связи вдвое больше и составляет $8 \mathrm{eV}$, то энергия, необходимая для освобождения иона внутри кристаллической решетки и обеспечения его миграции, оказывается приблизительно в 4-5 раз ниже [11] и составляет несколько менее $1 \mathrm{eV}$.

В то же время тепловая энергия ФП типа $\alpha \rightarrow \beta$ при температуре $T_{c}=176.3^{\circ} \mathrm{C}(449.3 \mathrm{~K})$ составляет
$40 \cdot 10^{-3} \mathrm{eV}$, что не может обеспечить переброс достаточного для разрыва окта-Ag-S- $\sigma$-связей количества электронов из валентной зоны в зону проводимости через щель $0.9 \mathrm{eV}$ (электроны при перебросе черпаются из валентной зоны, в которую расширяется энергетический уровень связывающей молекулярной орбитали, соответствующий окта-Ag-S- $\sigma$-связи).

Тем не менее эксперимент показывает, что переброс электронов в количестве, нужном для освобождения и миграции ионов окта- $\mathrm{Ag}^{+}$, происходит. Причиной такого положения являются корреляционные свойства соединения $\mathrm{Ag}_{2} \mathrm{~S}$, унаследовавшего от атома $\mathrm{Ag}$ способность понижать энергетическое положение зон при их заселенности электронами (см. выше). А именно, в сильно коррелированном соединении переброс даже небольшого количества электронов в зону проводимости приводит к ее понижению по энергии при одновременном подъеме по энергии ей навстречу опустошаемой валентной зоны. Сближение зон эффективно повышает заселенность зоны проводимости, что, в свою очередь, увеличивает переброс и т.д.

Поскольку электроны выкачиваются из связывающей (но не разрыхляющей) орбитали, соответствующей окта$\mathrm{Ag}-\mathrm{S}-\sigma$-связи и расширенной в кристалле до валентной зоны, то при достижении критической концентрации разорванных окта-Ag-S- $\sigma$-связей все окта-Ag-ионы скачкообразно получают возможность мигрировать подобно жидкости по решетке, а элементарная ячейка меняет свою симметрию с моноклинной на кубическую.

Таким образом, скачкообразному ФП предшествует протяженное по температуре корреляционное уменьшение ширины запрещенной зоны. Такой процесс в литературе обозначается как протяженный по температуре корреляционный переход Мотта [13], который инициирует структурный суперионный ФП. Такова предлагаемая в настоящей работе схема комплексного ФП полупроводник-суперионик.

\section{5. Заключение}

В работе исследованы температурные зависимости диэлектрических спектров пленок $\mathrm{Ag}_{2} \mathrm{~S}$ при расположении электродов в планарной геометрии (вдоль поверхности пленки). Предложен макроскопический механизм частотной зависимости электрического отклика планарной пленочной структуры на базе $\mathrm{Ag}_{2} \mathrm{~S}$, включающий эквивалентную электрическую схему планарной пленочной структуры. Предложена качественная микроскопическая модель совершения термического суперионного ФП в пленках $\mathrm{Ag}_{2} \mathrm{~S}$. Модель включает выявление окта-координированных ионов $\mathrm{Ag}^{+}$как основных претендентов на составные элементы „расплавленной“ части решетки в процессе ионной проводимости высокотемпературной фазы и выявление тетра-координированных ионов $\mathrm{Ag}$ как основных претендентов на составные элементы „нерасплавленной“ части решетки, стабилизирующей 
кристаллическую структуру в процессе ионной проводимости высокотемпературной фазы.

Таким образом, ДС позволяет выявлять не только макроскопические черты электрического отклика пленочной структуры на воздействие переменного электрического поля, но и создает условия для построения микроскопической картины механизмов фазовых превращений в пленках супериоников. Использованная в предлагаемой статье методика ДС и полученные результаты могут быть применены при исследовании пленок других материалов с суперионным ФП, таких как AgI, AgSe, AgTe или $\mathrm{Ag}_{2} \mathrm{GeS}_{3}$.

\section{Конфликт интересов}

Авторы заявляют, что у них нет конфликта интересов.

\section{Список литературы}

[1] Zhenwei Wang, Kaifa Luo, Jianzhou Zhao, Rui Yu. Phys. Rev. B 100, 205117-1 (2019).

[2] Z.K. Liu, J. Jiang, B. Zhou, Z.J. Wang, Y. Zhang, H.M. Weng, D. Prabhakaran, S-K. Mo, H. Peng, T. Kim, M. Hoesch, Z. Fang, X. Dai, Z.X. Shen, D.L. Feng, P. Dudin, ZY.L. Chen. Nature Mater. 13, 677 (2014)

[3] Е.А. Укше, Н.Г. Букун. Твердые электролиты. Наука, М (1977). $176 \mathrm{c}$.

[4] Jun Liu, Lu Chen, Hangsheng Yang, Ze Zhang, Yong Wang. Prog. Natur. Sci.: Mater. Int. 29, 397 (2019).

[5] O. Alekperov, O. Samedov, R. Paucar, N. Abdulzade, E. Nakhmedov, A. Nadjafov, K. Wakita, N. Mamedov. Phys. Status Solidi C 12, 6, 610 (2015).

[6] Sh. Miyatani. J. Phys. Soc. Jpn. 15, 9, 1586 (1960).

[7] S.I. Sadovnikov, A.I. Gusev, A.A. Rempel. Dokl. Phys. Chem. 464, 2, 238 (2015).

[8] Г.В. Самсонов, С.В. Дроздова. Сульфиды. М. (1972). C. $38-41$.

[9] Дж. Маррелл, С. Кеттл, Дж. Теддер. Теория валентности / Пер. с англ. под ред. М.Г. Веселова. Мир, М. (1968). 520 с.

[10] Г.Г. Филиппов, А.И. Горбунов. Рос. хим. журн. 39, 2. 39 (1995).

[11] Л. Цюлике. Квантовая химия. Мир, М. (1976). 512 с.

[12] Д.И. Блецкан, В.В. Вакульчак, А.В. Лукач, И.П. Студеняк. Науч. вестн. Ужгородского ун-та. Сер. физ. 40, 30 (2016).

[13] А.В. Ильинский, О.Е. Квашенкина, Е.Б. Шадрин. ФТП 45, 9, 1197, (2011).

Редактор Т.Н. Василевская 